ISSN 2413-0877 Volume 2 (2015) 70-77

The 3rd International Conference on Biological Science 2013

(The 3rd ICBS-2013)

\title{
HEPARANASE EXPRESSION IN RENAL INTERSTITIAL MAY CONTRIBUTE TO EPITHELIAL AND ENDOTHELIAL CELLS INJURIES AFTER KIDNEY ISCHEMIC/ REPERFUSION EPISODE IN MICE
}

\author{
Nur Arfian'1, Dwi C. Ratna Sari', Muhammad M. Romi', Dian P.Wibisono', \\ and Noriaki Emoto 2,3 \\ ${ }^{1}$ Anatomy, Embryology and Anthropology Department, Faculty of Medicine, Gadjah Mada University, \\ Jogjakarta, Indonesia \\ ${ }^{2}$ Clinical Pharmacy Department, Kobe Pharmaceutical University, Kobe, Japan \\ ${ }^{3}$ Cardiovascular Medicine Division, Internal Medicine Department, \\ Graduate School of Medicine Kobe University, Kobe, Japan \\ Email address: n_arfian@yahoo.com
}

\begin{abstract}
Kidney ischemia/reperfusion injury $(\mathrm{I} / \mathrm{R})$ is the most frequent cause of acute kidney injury (AKI). It had been reported that epithelial and endothelial injuries occurred during kidney I/R injury. Heparanase is an enzyme that degrades glycocalyx and contributes to I/R injury in the heart and liver. This study is to elucidate the association between heparanase expression and cell injuries in kidney I/R injury. We performed kidney $\mathrm{I} / \mathrm{R}$ injury model in mice using renal pedicle clamping for 30 minutes and sacrificed the mice in day $1(n=6)$ after operation. Sham-operation procedure $(\mathrm{SO}, \mathrm{n}=5)$ was used as control. PAS staining was used to quantify tubular injury score. Serum creatinine was measured from orbital venous. Heparanase expression was quantified using western blot and real-time PCR. Heparanase localization and endothelial injury were shown by immunostaining of heparanase and double glycocalyx-von Willebrand factor. Kidney I/R induced an increase of serum creatinine level that was accompanied by elevation of tubular injury score and glycocalyx damage. Glycocalyx damage was identified using immunofluorescent staining that revealed a disruption of glycocalyx or lectin layer in the endothelial cells of intra-renal artery. This finding was associated with significant elevation of heparanase mRNA and protein level expression. We found that heparanase was expressed in the renal epithelial and interstitial cells. In conclusion, heparanase may induce endothelial and epithelial injury in the kidney I/R episode. Using heparanase expression as a early marker of AKI may possibly promising.
\end{abstract}

Key words: kidney I/R, epithelial injury, endothelial injury, heparanase, glycocalyx

\section{INTRODUCTION}

Acute Kidney Injury (AKI) is the most common cause of mortality in clinical setting, especially in Intensive Care Unit (ICU) and elderly (Schrier et al., 20041). Kidney IschemiaReperfusion (I/R) injury is the most frequent cause of AKIdue to lack of oxygen supply to kidney, and $30-40 \%$ of this case lead to chronic kidney diseases (CKD) (Bonventre \& Yang, 2011). Epithelial cell injury plays a crucial role in the AKI, and it is worsen by endothelial cell injury. Endothelial cell injury induces prolongation of epithelial injury (Molitoris et al., 2002).

Endothelial cell injury that occurs in the extension phase of kidney $\mathrm{l} / \mathrm{R}$ leads to epithelial injury and inflammation (Molitoris \& Sutton, 2004; Sutton et al., 2002). Endothelial cell injury includes morphological changing and actin protein changing (Sutton et al., 2003). Previous result showed swelling of endothelial cells, disruption of 2 endothelial cell attachment and endothelial cell membrane changing occurred during l/R injury (Brodsky et al., 2002). Hipoxic condition in the heart and liver caused disruption of glycocalyx, an endothelial surface layer that consist of lectin and proteoglycan (Beresewicz et al., 1998). In CKD, disruption and damage of glycocalyx had been reported in albuminuria (Salmon \& Satchell, 2012), and

ISSN 2413-0877 @ 2015 The Authors.

Published by KnowledgeE Publishing Services This is an open access article under the CC BY-NC-ND license (http://creativecommons.org/licenses/by-nc-nd/4.0)

Selection and Peer-review under responsibility of the 3rd ICBS-2013

Doi http://dx.doi.org/10.18502/kls.v2i1.119 
adryamicin induced nephropathy (Kramer et al., 2006).

Glycocalyx is composed of glycosaminoglycan (GAG) component that consists of heparansulphate (HS) and chondroitin sulphate. Heparan sulphate (HS) is the main component of GAG that arranges $40-50 \%$ of glycocalyx (Mulivor \& Lipowsky, 2004). In this study, we want to elucidate the early event of glycocalyx disruption and the expression of heparanase in the kidney $\mathrm{l} / \mathrm{R}$ condition that may associates with eNOS expression. Heparanase is an endoglycosidase enzyme that functions to cleaveheparansulphate (HS). Heparanase also may provide a property for the early marker of AKI.

\section{MATERIALS AND METHODS}

\section{Animal Experiment and Kidney Ischemic Reperfusion Injury Model in Black-six}

We performed kidney ischemic/reperfusion injury model $(\mathrm{I} / \mathrm{R})$ in mice, male blacksix background, 3-4 months old, 30 grams. The mice were housed in 12-hr light and dark cycle with free access of water and chow. Four-month old male mice (each $n=8)$ littermates were subjected to kidney Ischemic Reperfusion Injury (IRI) model. Experiments were conducted by following established guideline for animal cares of Gadjah Mada University. The surgical preparation and operation used based on previously described methods (Wu et al., 2007). Briefly, mice were anesthetized using inhalational isofluran (Merck). Abdomen was opened and both renal pedicles were clamped using non-traumatic microaneurysm clamps for 30 minutes. Mice were placed in 370 heating pad during ischemic period. After removal of clamp, abdomen was closed. SO mice $(n=5)$ underwent similar procedure except for renal pedicles clamping. We sacrificed the mice in day 1 after operation to examine the extension phase of IRI. Blood was collected from Scleral Venous and kidney tissues were divided into snap frozen for RNA and protein examinations, embedded in OCT compound and fixed in $4 \%$ PFA for paraffin.

\section{Kidney Function Assessment}

Serum creatinin was measured in the Clinical Pathology Department of Medical Faculty Gadjah Mada University.

\section{Histological analysis}

Four-mm paraffin sections were de-paraffinized, heated in Citrate Buffer (except for ET1 antibody staining) and stained for Periodic Acid Schiff to evaluate tubular injury and immunostaining. Immunohistochemical stainings were done for anti-Heparanase antibody 3 (Abcam). Sections were de-paraffinized, and then incubated in 3\% $\mathrm{H} 2 \mathrm{O} 2$. Antibodies were incubated for overnight. Secondary antibody incubation was done for 1 hour using Anti Rabbit Dako Envision System HRP labeled Polymer. Double immunofluorescencestainings were done for von-Willebrand (DAKO) and Wheat Germ Aglutinin (WGA)-AF 488 conjugated (Invitrogen).

\section{Tubular Injury Score}

Tubular injury was scored based on PAS staining. Scoring was done with grading tubular injury, epithelial cell apoptosis, intra-luminal cash and brush border loss in 15 randomly 
chosen, non-overlapping fields ( 200 magnification). The lesions were graded on a scale from 0 to $4: 0=$ normal; 1 = mild, involving less than $25 \%$ of the cortex; $2=$ moderate, involving 25 to $50 \%$ of the cortex; $3=$ severe, involving 50 to $75 \%$ of the cortex; and $4=$ extensive damage, involving more than $75 \%$ of the cortex (Inazaki et al., 2004).

\section{Real-time PCR}

RNA was isolated from kidney tissue using Trizol (Invitrogen). cDNA was synthesized using ReverTra (TOYOBO Co;Ltd, TRT-101x10). Quantitative real-time PCR was performed

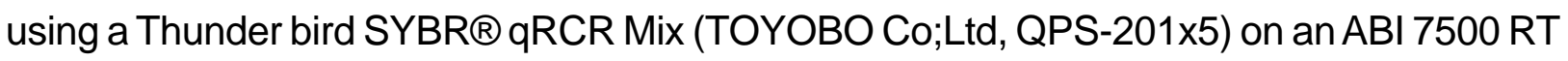
thermocycler, and an ABI 7500 RT PCR System.

\section{Western blotting}

Proteins extracted from kidney tissues were separated on SDS-PAGE, transferred to a polyvinylidene fluoride (PVDF) transfer membrane, and probed with E-cadherin polyclonal antibody (ABCAM), eNOS (BD Pharmingen), SOD2 (Santa Cruz), ETAR (Santa Cruz), PKC (Santa Cruz) and GAPDH antibody (Invitrogen). Proteins were visualized using an ECL Plus WB detection system (Amersham-GE Healthcare). Blots were quantified by densitometry using LSI.

\section{Hypoxic endothelial cells in in-vitro experiment}

We induced hypoxic condition for 30 minutes in the MS-1 endothelial cells culture using hypoxic bag. Briefly, MS-1 cells were cultured in the $10 \mathrm{~cm}$ dish. Hypoxic bags with oxygen absorbent were prepared and waited to reach $0 \%$ oxygen content. Then, we put the dish in the hypoxic bag for 30 minute. After that, we extracted RNAusing Trizol and quantified prepro-ET1, heparanase, endothelial Nitrite Oxide Synthase (eNOS) and ICAM-1.

\section{RESULTS AND DISCUSSION}

\section{Increase of kidney injury and tubular injury after Ischemic-Reperfusion Episode}

Serum creatinine level increased dramatically during 24 hour after kidney I/R injury (Fig. 1A). It demonstrated rapid disruption of kidney tubular epithelial cells occurred due to ischemic effect. Rapid elevation of creatinine level was followed by injury in tubular system with apoptosis of epithelial cells. Kidney I/R injury caused an increase of kidney injury and tubular injury score in cortex (Fig. 1 B).

\section{Glycocalyx disruption in intra-renal artery and reduction of eNOS expression}

We observed endothelial surface layer or glycocalyx in endothelial cell of intra-renal arteries using WGA and von-Willebrand Factor (endothelial cell marker). We found that kidney I/R induced disruption of glycocalyx as shown by un-complete staining of WGA in endothelial layer of intra-renal arteries (Fig.2A) after kidney I/R injury. However, we did not find the disruption of endothelial cell in these arteries. This finding might associated with reduction of eNOS expression, as shown in Figure 2B. 
A Serum creatinine $(\mathrm{mg} / \mathrm{dL})$
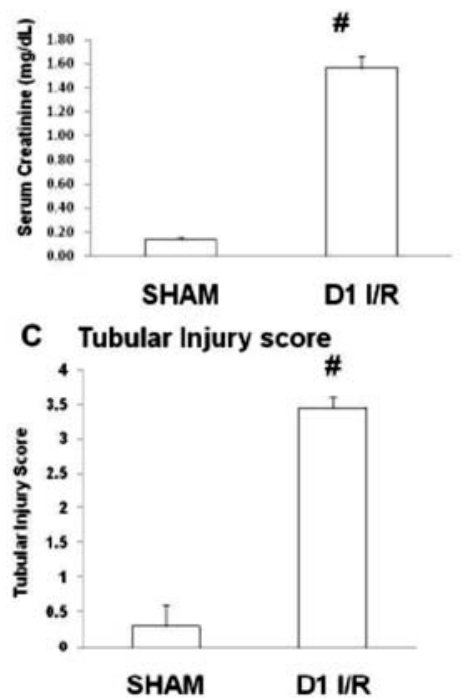

B
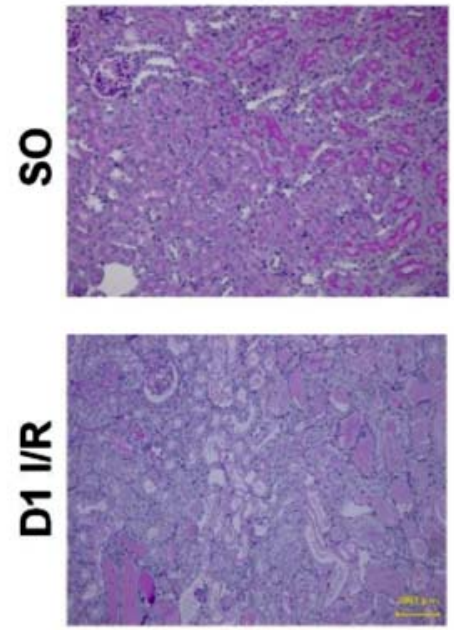

Figure1. Creatinine serum level and tubular injury score in day 1 after kidney I/R (A) Creatinine serum level in day 1 after kidney $\mathrm{I} / \mathrm{R}$ injury. Blood was taken from orbital venous from mice with kidney I/R injury (each $n=5)$ and SO $(n=4)(B-C)$ Representative picutures of PAS staining and tubular injury score quantification (200x magnification, 12-15 field each samples, $n=5)$. Data were presented as mean $\pm S D$. \# $P<0.001$ vs $S O$ group. Bar $=50 \mu \mathrm{m}$.

A
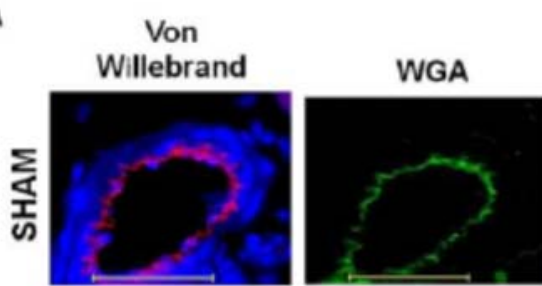

OVERLAY
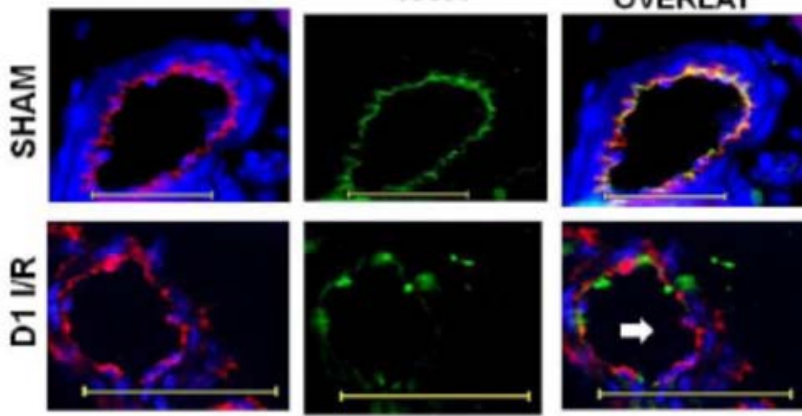

B

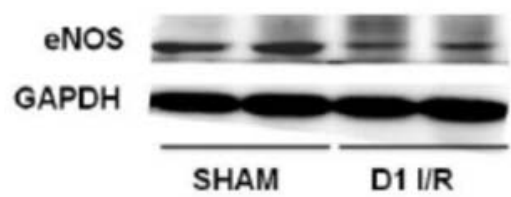

Figure2. (A) Immunostaining of WGA and von-Willebrand factor to see glycocalyx or endothelial surface layer and endothelial cells in the vessel. Arrow indicate disruption of glycocalyx, but not endothelial cells in day 1 after IRI. (B) eNOS expression after kidney I/R injury. Bar $=50 \mu \mathrm{m}$.

\section{Upregulation of Heparanase expression after kidney I/R in epithelial and interstitial cell}

To observe the basis of glycocalyx disruption, we quantified heparanase expression in kidney I/R injury. Kidney I/R injury induced an significant increase of heparanase mRNA and protein expression (Fig.3 A\&B). We did immunostaining of heparanase and revealed that epithelial cells and interstitial cells as sources of heparanase (Fig.3 C) 


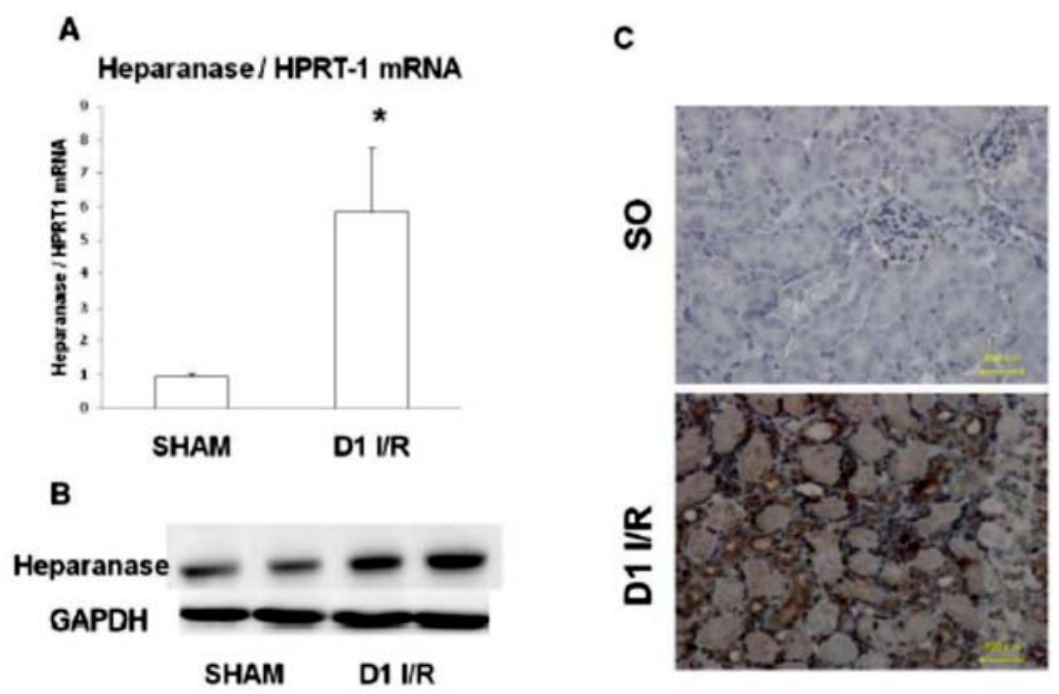

Figure3. Effects of kidney l/R injury to Heparanase expression. (A) mRNA level of Heparanase in SO and D1 I/R goup (each $n=4-5$ ). (B) Heparanase protein level after kidney I/R injury. (C) Heparanase was expressed in the renal interstitial space and epithelial cells. Data was presented as mean $\pm \mathrm{SD} .{ }^{*} \mathrm{P}<0.05$ vs sham. Bar $=50 \mu \mathrm{m}$.
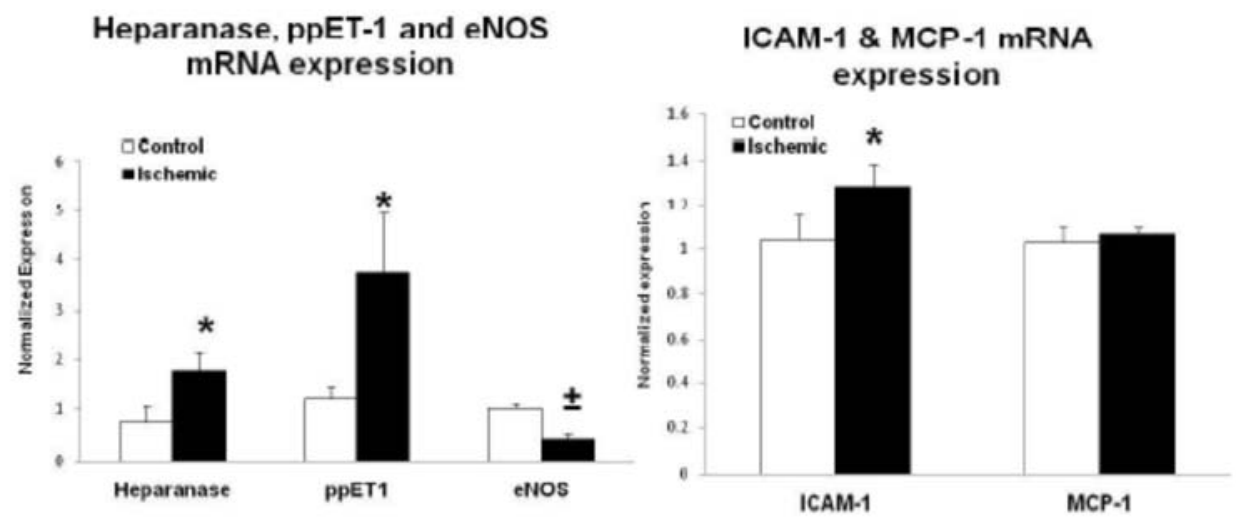

Figure 4. Effect of 30 minutes ischemia to heparanase, pre-pro ET-1, eNOS and inflammatory mediators mRNA level. (A) ischemic induced upregulation of heparanase and pre-pro ET-1 expression and down regulation of eNOS expression. (B) Ischemia also induced upregulation of inflammatory mediator, such as ICAM-1 but not MCP-1 in MS1 endothelial cell culture. Data were presented as mean $\pm \mathrm{SD}$. ${ }^{*} \mathrm{P}<0.05$ vs control. $\pm \mathrm{P}<0.01$ vs control.

Targeting early event of endothelial injury due to ischemic-reperfusion, in this present study we observed glycocalyx disruption in the extension phase of I/R injury. Shedding of glycocalyx is reported in patients undergoing major vascular surgery due to global and regional ischemic (Rehm et al., 2007). Using WGA staining in the vessel, we demonstrated disruption of glycocalyx in the intra-renal artery, without followed by endothelial cell damage. We concluded that glycocalyx disruption associated with endothelial dysfunction as shown by eNOS reduction (Fig.2). Until now, there are no consistent results about endothelial cells morphology changing during this phase, endothelial dysfunction is believed contributing in this phase. Actin cytoskeleton and junctional alteration have been already reported occurs in the vascular endothelial cell using Tie2/GFP following renal ischemic injury in vivo (Sutton et 
al., 2002; Sutton et al., 2003). Another studies showed morphologic changing of endothelial cell including endothelial cell swelling, endothelial cell to cell attachment disruption, and endothelial cell-basement membrane attachment alteration in the renal microvasculature (Brodsky et al., 2002).

Loss of glomerular endothelial glycocalyx in adriamycin nephropathy model associated with down regulation of $\mathrm{HS}$ and enzymes that play role in synthesis and elongation of proteoglycan GAG chains (Jeansson et al., 2009). HS loss is also induced by heparanase production, an endoglycosidase that degrades HS (Rao et al., 2011). In this study, we observed an increase of heparanase expression in extension phase of kidney l/R injury (Fig.3). The loss of HS is demonstrated in some glomerular diseases in several human and animal experimental studies, such as passive Heymann Nephritis, anti-GBM Nephritis, puromicyn induced nephrosis, adriamycin-induced nephrosis, and minimal change diseases. HS loss in those conditions are correlated with higher expression of glomerular heparanase (van den Hoven et al., 2007). Treatment of human heparanase and heparinase in cultured gomerular endothelial cells induces disruption of glycocalyx (Singh et al., 2007). An increase of heparanase also occurs after focal cerebral ischemic in the mouse brain and correlates with proliferating endothelial cells undergoing angiogenesis (Li et al., 2012). Heparanase up-regulation in endothelial cells and macrophages is suggested to play role in reduction of HS amount in arterial wall and contributes to atherosclerosis (Rao et al., 2011). It seem that high expression of heparanase after kidney $\mathrm{l} / \mathrm{R}$ injury leads to endothelial dysfunction with eNOS reduction and glycocalyx disruption.

We also showed epithelial cell and interstitial cells as sources of heparanase expression (Fig.3). We assumes that $\mathrm{I} / \mathrm{R}$ injury contributes to the expression of heparanase in those cells. Renal epithelial cell has been also shown as a source of heparanase under hyperglycemic condition (Maxhimer et al., 2005). Meanwhile, heparanase expression in interstitial cells might associate with ROS production. Previously, we reported that kidney $\mathrm{l} / \mathrm{R}$ induced ROS production that is inhibited by ET-1 deletion from endothelial cells (Arfian et al., 2012). Our results are consistent with several studies that reported about the role of ROS in mediating heparanase production, particularly in high glucose condition (Rao et al., 2011), as well as aldosteron and angiotensin II treatment in glomerulus (van den Hoven et al., 2009). Because of ROS has different role in the epithelial and interstitial cells after kidney I/R injury (Kim et al., 2010), it seem that heparanase also regulate those cells differently. For further study, it is interesting to elucidate role of heparanase in these different cells after kidney I/R injury.

In the kidney diseases, glycocalyx disruption and shedding have been already known occur in inflammation and ischemic condition (Mulivor \& Lipowsky, 2004; Lipowsky et al., 2011), albuminuria and microvascular permeability (Salmon \& Satchell, 2012) and adriamycin nephropathy (Jeansson et al., 2009). We found here in the culture of endothelial cell in hypoxic condition demonstrated an increase of inflammation mediator such as ICAM-1. This might associate with elevation of heparanase and pre-pro ET-1 level. It might show that hypoxic condition lead to up-regulation of heparanase in endothelial cells. Meanwhile, in the ischemic and reperfusion the others cells express heparanase, such as epithelial and interstitial cells. 
Taken together HS and glycocalyx loss after kidney I/R might be caused by higher expression of heparanase. Heparanase that is expressed not only by endothelial cells, but also by epithelial and interstitial cells might contribute to tubular injury and endothelial dysfunction in kidney $\mathrm{l} / \mathrm{R}$ injury.

\section{REFERENCES}

Arfian, N., N. Emoto, N. Vignon-Zellweger, and K. Nakayama. 2012. ET-1 deletion from endothelial cells protects the kidney during the extension phase of ischemia/reperfusion injury. Biochem Biophys Res Commun 425: 443-449.

Beresewicz, A., E. Czarnowska, and M. Maczewski. 1998. Ischemic preconditioning and superoxide dismutase protect against endothelial dysfunction and endothelium glycocalyx disruption in the postischemic guinea-pig hearts. Mol Cell Biochem 186: 87-97.

Bonventre, J.V., and L. Yang. 2011. Cellular pathophysiology of ischemic acute kidney injury. J Clin Invest 121: 4210-4221.

Brodsky, S.V., T. Yamamoto, T. Tada, and B. Kim. 2002. Endothelial dysfunction in ischemic acute renal failure: rescue by transplanted endothelial cells. Am J Physiol Renal Physiol 282: F1140-1149.

Inazaki, K., Y. Kanamaru, Y. Kojima, and N. Sueyoshi. 2004. Smad3 deficiency attenuates renal fibrosis, inflammation, and apoptosis after unilateral ureteral obstruction. Kidney Int 66: 597-604.

Jeansson, M., K. Bjorck, O. Tenstad, and B. Haraldsson. 2009. Adriamycin alters glomerular endothelium to induce proteinuria. J Am Soc Nephrol 20: 114-122.

Kim, J., K.J. Jung, and K.M. Park. 2010. Reactive oxygen species differently regulate renal tubular epithelial and interstitial cell proliferation after ischemia and reperfusion injury. Am J Physiol Renal Physiol 298: F1118-1129.

Kisanuki, Y.Y., N. Emoto, T. Ohuchi, and B. Widyantoro. 2011. Low blood pressure in endothelial cell-specific endothelin 1 knockout mice. Hypertension 56: 121-128.

Kramer, A., M. van den Hoven, A. Rops, and T. Wijnhoven. 2006. Induction of glomerular heparanase expression in rats with adriamycin nephropathy is regulated by reactive oxygen species and the renin-angiotensin system. J Am Soc Nephrol 17: 2513-2520.

Li, J., J.P. Li, X. Zhang, Z. Lu. 2012. Expression of heparanase in vascular cells and astrocytes of the mouse brain after focal cerebral ischemia. Brain Res 1433: 137-144.

Lipowsky, H.H., L. Gao, and A. Lescanic. 2011. Shedding of the endothelial glycocalyx in arterioles, capillaries, and venules and its effect on capillary hemodynamics during inflammation. Am J Physiol Heart Circ Physiol 301: H2235-2245.

Maxhimer, J.B., M. Somenek, G. Rao, C.E. Pesce. 2005. Heparanase-1 gene expression and regulation by high glucose in renal epithelial cells: a potential role in the pathogenesis of proteinuria in diabetic patients. Diabetes 54: 2172-2178.

Molitoris, B.A., R. Sandoval, and T.A. Sutton. 2002. Endothelial injury and dysfunction in ischemic acute renal failure. Crit Care Med 30: S235-240.

Molitoris, B.A., and T.A. Sutton. 2004. Endothelial injury and dysfunction: role in the extension phase of acute renal failure. Kidney Int 66: 496-499. 
Mulivor, A.W., and H.H. Lipowsky. 2004. Inflammation-and ischemia-induced shedding of venular glycocalyx. Am J Physiol Heart Circ Physiol 286: H1672-1680.

Rao, G., H.G. Ding, W. Huang, and D. Le. 2011. Reactive oxygen species mediate high glucose-induced heparanase-1 production and heparan sulphate proteoglycan degradation in human and rat endothelial cells: a potential role in the pathogenesis of atherosclerosis. Diabetologia 54: 1527-1538.

Rehm, M., D. Bruegger, F. Christ, and P. Conzen. 2007. Shedding of the endothelial glycocalyx in patients undergoing major vascular surgery with global and regional ischemia. Circulation 116: 1896-1906.

Salmon, A.H., and S.C. Satchell. 2012. Endothelial glycocalyx dysfunction in disease: albuminuria and increased microvascular permeability. J Pathol226: 562-574.

Schrier R.W., W. Wang, B. Poole, and A. Mitra. 2004. Acute renal failure: definitions, diagnosis, pathogenesis, and therapy. J Clin Invest 114: 5-14.

Singh, A., S.C. Satchell, C.R. Neal, E.A. McKenzie. 2007. Glomerular endothelial glycocalyx constitutes a barrier to protein permeability. J Am Soc Nephrol 18: 2885-2893.

Sutton, T.A., C.J. Fisher, and B.A. Molitoris. 2002. Microvascular endothelial injury and dysfunction during ischemic acute renal failure. Kidney Int 62: 1539-1549.

Sutton, T.A., H.E. Mang, S.B. Campos, and R.M. Sandoval. 2003. Injury of the renal microvascular endothelium alters barrier function after ischemia. Am J Physiol Renal Physiol 285: F191-198.

van den Hoven, M.J., A.L. Rops, I. Vlodavsky, V. Levidiotis. 2007. Heparanase in glomerular diseases. Kidney Int 72: 543-548.

van den Hoven, M.J., F. Waanders, A.L. Rops, and A.B. Kramer AB. 2009. Regulation of glomerular heparanase expression by aldosterone, angiotensin II and reactive oxygen species. Nephrol Dial Transplant 2009; 24: 2637-2645.

Wu, H., G. Chen, K.R. Wyburn, and J. Yin. 2007. TLR4 activation mediates kidney ischemia/ reperfusion injury. J Clin Invest 117: 2847-2859. 9 